\title{
Dos alimentos cardioprotetores ao padrão alimentar: uma revisão da literatura
}

\author{
Mariana R. C. Portugal, ${ }^{1}$ Ana Carolina Gonçalves, ${ }^{2}$ Grazielle V. B. Huguenin, ${ }^{3}$ Annie S. B. Moreira ${ }^{3 *}$
}

\section{Resumo}

Apesar de muitas revisões terem focado em alimentos e nutrientes como determinantes da doença cardiovascular (DCV), pouco se sabe sobre o papel do padrão alimentar e do consumo de alimentos ultraprocessados. O objetivo desta foi abordar os padrões alimentares cardioprotetores mais estudados, alimentos considerados importantes à saúde cardiovascular e o crescente consumo de alimentos ultraprocessados e sua relação com as doenças cardiovasculares.Um padrão alimentar baseado em grupos de alimentos com ação cardioprotetora mostra-se mais benéfico do que a suplementação, por exemplo, dos nutrientes isolados. Isso pode ser explicado pelo sinergismo entre os nutrientes, aumentando a efetividade. Além da relevância do padrão alimentar, o foco cada vez mais é fazer dos alimentos in natura ou minimamente processados a base da alimentação, uma vez que são alimentos naturalmente ricos em nutrientes como fibras, vitaminas, antioxidantes, minerais, ácidos graxos mono e poli-insaturados, e pobres em carboidratos simples, gordura trans, sódio e aditivos químicos. Dentro deste contexto, desestimular o consumo dos alimentos ultraprocessados também é fundamental para a prevenção de doenças crônico-degenerativas, bem como para a preservação de padrões alimentares tradicionais.

Descritores: Cardiopatias; Hábitos alimentares; Indústria de processamento de alimentos.

\section{Abstract \\ From cardioprotective foods to dietary pattern: A literature review}

Although many reviews have focused on food and nutrients as determinants of cardiovascular disease (CVD), little is known about the role of dietary pattern and consumption of ultra-processed food products. The present report intended to examine the most studied dietary patterns, foods considered important to cardiovascular health, the increasing consumption of ultra-processed food products and its relation to cardiovascular diseases. A food pattern based on food groups with cardioprotective effect was shown to be more beneficial than supplementation, for example, of isolated nutrients. This can be explained by the synergism between the nutrients, increasing their effectiveness. In addition to the importance of the dietary pattern, the focus is to increase the ingestion of in natura and minimally processed foods, once they are higher in important nutrients such as fiber, vitamins, antioxidants, minerals, mono and polyunsaturated fatty acids, and lower in refined carbohydrates, trans fat, sodium and chemical additives. In this context, discourage the consumption of ul-
1. Programa de pós-graduação em Alimentação, Nutrição e Saúde. Instituto de Nutrição. Universidade do Estado do Rio de Janeiro. Rio de Janeiro, RJ, Brasil.

2. Instituto de Nutrição. Universidade do Estado do Rio de Janeiro. Rio de Janeiro, RJ, Brasil.

3. Departamento de Nutrição Aplicada. Instituto de Nutrição. Universidade do Estado do Rio de Janeiro. Rio de Janeiro, RJ, Brasil.

\section{*Endereço para correspondência:}

São Francisco Xavier, 524, 12ㅇandar

Rio de Janeiro, RJ, Brasil. CEP: 20550-900

E-mail: anniebello@gmail.com

Revista HUPE, Rio de Janeiro, 2015;14(3):27-33

doi: 10.12957/rhupe.2015.19873

Recebido em 19/03/2015. Aprovado em 02/07/2015.

tra-processed food products is also essential for the prevention of chronic degenerative diseases as well as for the preservation of traditional dietary patterns.

Keywords: Heart diseases; Food habits; Food-processing industry.

\section{Resumen}

\section{Alimentos cardioprotectores en el patrón alimen- tario: una revisión de la literatura}

Aunque muchas revisiones se han centrado en los alimentos y nutrientes como factores determinantes de las enfermedades cardiovasculares (ECV), poco se sabe sobre el papel del patrón alimentario y el consumo de alimentos ultra-procesados. El objetivo de esta revisión fue abordar los patrones alimentares cardioprotectores más estudiados, alimentos que son considerados importantes para la salud cardiovascular y el creciente consumo de alimentos ultra-procesados y su relación con las enfermedades cardiovasculares. Un patrón alimentario basado en grupos de alimentos con acción cardioprotectora demostró ser más beneficioso que la administración de suplementos, por ejemplo, de nutrientes aislados. Esto puede explicarse por el sinergismo entre los nutrientes, aumentando la efectividad. Además de la importancia del patrón alimentario, el objetivo es hacer progresivamente de los alimentos naturales o mínimamente procesados, la base de la alimentación, porque son alimentos naturalmente ricos en nutrientes como fibra, vitaminas, antioxidantes, minerales, ácidos grasos mono y poliinsaturados, y bajos en carbohidratos simples, grasas trans, sodio y aditivos químicos. En este contexto, desalentar 


\section{Artigo de revisão}

el consumo de alimentos ultra-procesados es también esencial para la prevención de enfermedades crónico- degenerativas, así como para la preservación de los patrones de alimentación

\section{Introdução}

A doença cardiovascular (DCV) está entre as principais causas de morte no mundo, sendo responsável por mais de 17 milhões de óbitos no ano de 2008. Deste total, mais de 3 milhões ocorreram antes dos 60 anos de idade e poderiam ser amplamente prevenidas. ${ }^{1}$ No Brasil, as DCV são as principais causas de morte em homens e mulheres, sendo responsáveis por cerca de $20 \%$ do total de óbitos em indivíduos acima de 30 anos. ${ }^{2}$ A OMS estima que $75 \%$ da mortalidade cardiovascular podem ser diminuídos com adequadas mudanças no estilo de vida e esse é o grande desafio das diversas diretrizes existentes em prevenção cardiovascular atualmente. ${ }^{3}$

Um dos primeiros indícios de que este grupo de patologias é influenciado pela nutrição surgiu a partir da observação feita por Ignatowski em 1908. Segundo ele, uma alta ingestão de alimentos fontes de colesterol, como ovos, carne e leite integral, promovia aterosclerose em coelhos. ${ }^{4}$ A partir daí, iniciou-se um extenso período de estudos a respeito da fisiopatologia da aterosclerose e o papel que a alimentação exerce neste processo. Uma das primeiras organizações a apoiar as mudanças dietéticas foi a American Heart Association (AHA), a Associação Americana do Coração. ${ }^{5}$ Em 1957, o Comitê de Nutrição da AHA em cooperação com a Sociedade para o Estudo da Arteroesclerose (Society for the Study of Arteriosclerosis) concluíram que a dieta exerce um papel fundamental na patogênese da aterosclerose. A dieta deveria ser balanceada e variada, com ajuste do total calórico realizado para o alcance ou manutenção do peso desejado. Além disso, houve ainda o estímulo à substituição da gordura de origem animal pelas poli-insaturadas, com total de gorduras fornecendo de $25 \%$ a $30 \%$ das calorias. Até então, as recomendações tinham como foco principal os macronutrientes, especificamente as gorduras. ${ }^{6}$

Ao longo dos anos 60, as diretrizes foram revistas e pessoas de todas as idades passaram a ser estimuladas a limitar o colesterol dietético. A partir dos anos 80, elas passaram a se basear em conceitos mais sólidos, com grande incentivo ao consumo de frutas, vegetais, oleaginosas, limite ao consumo de carne vermelha e laticínios com alto teor de gordura, maior consumo de peixes, aves, redução do sal e açúcar, uso do azeite de oliva e outros óleos vegetais, e a substituição dos grãos tradicionales.

Palabras clave: Cardiopatías; Hábitos Alimenticios; Industria de procesamiento de alimentos.

refinados pelos integrais. Atualmente, o grande foco das pesquisas é interação entre os diferentes alimentos que constituem a dieta e não apenas o estudo dos efeitos dos nutrientes isolados. ${ }^{7}$ Esta revisão tem como objetivos principais (a) definir as características de padrões alimentares cardioprotetores mais estudados; (b) abordar alguns alimentos considerados importantes à saúde cardiovascular; (c) discutir o papel dos alimentos ultraprocessados na patogênese das doenças cardiovasculares e no surgimento de um padrão alimentar globalizado.

\section{Padrão alimentar cardioprotetor}

Historicamente, o papel de nutrientes específicos tem sido o foco principal na prevenção e tratamento das DCVs, entretanto, cada vez mais atenção tem sido direcionada ao padrão alimentar e a sua relação com saúde. ${ }^{8}$ Dentro deste panorama, a dieta DASH (Dietary Approaches to Stop Hypertension) e a dieta do Mediterrâneo são as mais estudadas em relação aos seus diversos efeitos sobre a saúde cardiovascular e efeitos sobre a saúde em geral. ${ }^{9}$ A primeira é reconhecida por sua ação na redução da pressão arterial e também pode reduzir de maneira significativa o risco para DCVs. ${ }^{10}$ Por sua vez, desde 1995, a associação entre a Dieta do Mediterrâneo e razão mortalidade coronariana/mortalidade total tem sido avaliada em diversos estudos e os resultados apontam para os efeitos benéficos. ${ }^{11}$

Diversos padrões alimentares considerados saudáveis já foram estudados e todos compartilham algumas características em comum, incluindo a ênfase ao consumo de frutas, hortaliças e outros alimentos de origem vegetal como oleaginosas e grãos integrais, maior consumo de peixes, ingestão limitada ou ocasional de laticínios, bem como de carne vermelha ou carnes processadas, redução na ingestão de carboidratos refinados e outros processados. As dietas vegetariana e japonesa também compartilham algumas características positivas associadas à saúde cardiovascular. Entretanto, como há menos evidências epidemiológicas e clínicas em relação aos seus benefícios, a importância de ambas é bastante limitada em relação aos padrões DASH e Mediterrâneo. ${ }^{9}$

A dieta DASH tem sido recomendada para a prevenção e tratamento das DCVs em importantes diretrizes 
internacionais, como AHA/ACC Lifestyle Management, e pela ACC/AHA Blood Cholesterol Guideline.12 A dieta caracteriza-se por estimular o consumo de vegetais e frutas, bem como de leite e derivados com baixo teor de gordura, grãos integrais, aves, peixes e oleaginosas. Por outro lado, limita a ingestão de gordura saturada, carne vermelha, doces e bebidas contendo açúcar. Em comparação às dietas usuais, a DASH fornece grandes quantidades de potássio, cálcio, magnésio, fibra e proteína. Seguir este padrão alimentar pode reduzir de maneira significativa o risco de doenças cardiovasculares, derrame e falência cardíaca em 20\%, 19\% e 29\%, respectivamente. ${ }^{10}$

Em relação à dieta do Mediterrâneo, estudos como o Lyon Diet Heart Study $y^{13}$ e HALE $^{14}$, assim como os americanos NIH-AARP Diet and Health Study ${ }^{15}$ e o Nurses Health Stud $y^{16}$ sugerem que um alto grau de adesão à dieta do Mediterrâneo está associado à redução tanto da mortalidade total quanto da mortalidade por DCV. O estilo de vida do Mediterrâneo é adotado por diferentes países ao longo do mar Mediterrâneo. Trata-se de um perfil alimentar caracterizado pelo alto consumo de frutas, hortaliças, leguminosas, carboidratos complexos, laticínios na forma de queijos e iogurte, consumo de azeite de oliva como principal fonte de lipídeos da dieta e ingestão moderada de vinho tinto junto às refeições. ${ }^{17}$ Recentemente, na Espanha, o ensaio clínico multicêntrico PREDIMED (Prevención con Dieta Mediterránea) demonstrou os efeitos positivos do padrão alimentar mediterrâneo na redução da mortalidade entre indivíduos com alto risco cardiovascular. ${ }^{18}$

Além das questões inerentes à composição da dieta, os aspectos culturais e o estilo de vida são fundamentais para que todos os benefícios sejam obtidos a partir deste padrão alimentar. O estilo de vida mediterrâneo preconiza aspectos importantes como comer em porções moderadas, realizar atividades culinárias, descansar por um pequeno período após as refeições, dar preferência aos alimentos sazonais, frescos e minimamente processados, bem como praticar alguma atividade física regularmente. ${ }^{19}$

Embora considerado saudável, tal padrão alimentar pode ter baixa adesão em países fora do Mediterrâneo. ${ }^{20}$ Em 2012, Weber e colaboradores publicaram os resultados do estudo piloto com uma dieta cardioprotetora brasileira conduzido no Hospital do Coração (HCOR) em SP. Um dos objetivos do trabalho era adaptar a dieta do Mediterrâneo estimulando o consumo de alimentos regionais e acessíveis à boa parte da nossa população. $\mathrm{O}$ ensaio clínico foi realizado com pacientes em preven- ção secundária para DCV, os quais foram acompanhados durante 12 semanas. Aqueles que faziam parte do grupo da dieta cardioprotetora brasileira se beneficiaram com maiores reduções na pressão arterial, glicemia e IMC, quando comparados àqueles que receberam orientações gerais conforme as diretrizes brasileiras para doenças cardiovasculares. ${ }^{21}$ Tais resultados foram atribuídos principalmente à inclusão de alimentos amplamente disponíveis em nosso país e evidencia que adaptação do padrão alimentar mediterrâneo se faz necessária, especialmente em locais onde a incidência das doenças cardiovasculares cresce cada vez mais.

\section{Alimentos cardioprotetores}

Certos alimentos são compostos por nutrientes que são benéficos para a saúde do coração, destacando-se os peixes, os vegetais, as frutas e as castanhas.

\section{Peixes}

Peixes e outros frutos do mar contêm diversos componentes que são benéficos para a saúde, incluindo algumas proteínas específicas, ácidos graxos insaturados, principalmente ácidos graxos poli-insaturados da série $\mathrm{n}-3$, vitamina $\mathrm{D}$ e selênio. ${ }^{9}$

Peixes como anchova, arenque, salmão selvagem ou de criação, sardinha, truta e atum branco tendem a apresentar maiores concentrações de ácidos graxos poli-insaturados..$^{22}$ Ácidos graxos poli-insaturados da série n-3 são considerados os nutrientes chaves, que supostamente seriam os responsáveis pelo potencial efeito cardioprotetor. ${ }^{23}$

Alguns trabalhos foram publicados mostrando associação inversa entre consumo de peixe e seus nutrientes chaves e DCV. Um deles é o estudoJELIS (2007), o qual sugere que pacientes com história de doença arterial coronariana que receberam tratamento com EPA apresentaram melhora de eventos cardiovasculares em $19 \%$, mostrando que este tratamento é promissor no que tange a prevenção de eventos cardiovasculares. ${ }^{24} \mathrm{Em}$ contrapartida, outros estudos que buscaram analisar os efeitos da suplementação de ômega 3 não obtiveram resultados consistentes para a prevenção de DCV. Conforme metanálise publicada em 2012, a suplementação com ômega 3 não apresenta associação significativa com mortalidade geral, morte cardíaca, morte súbita , infarto ou acidente vascular cerebral. ${ }^{25}$

Na maioria dos estudos experimentais que buscam investigar os efeitos dos ácidos graxos poli-insaturados da série n-3 sobre o risco cardiovascular, as doses utilizadas dos mesmos apresentavam-se em maior quantidade 


\section{Artigo de revisão}

do que aquelas tipicamente encontradas na dieta. ${ }^{26} \mathrm{Em}$ contraste, benefícios para a saúde cardiovascular, a partir do consumo de quantidade moderada de peixe, foram observadas em diferentes estudos. ${ }^{23,27}$

As proteínas dos peixes contêm arginina e glutamina, que são conhecidas por regular a função cardiovascular. Os peixes contêm ainda alguns elementos traço como o selênio e o cálcio que, direta ou indiretamente, promovem benefícios para a saúde cardiovascular, seja de forma isolada ou em combinação com os ácidos graxos poli-insaturados da série n-3.28 Embora mais estudos sejam necessários, é plausível que as interações entre ácidos graxos poli-insaturados da série n-3 e outros nutrientes, incluindo elementos traço, vitaminas e aminoácidos encontrados nos peixes desempenhem um papel importante na redução do risco de doenças cardiovasculares com uma atuação em conjunto. Portanto, é provável que o consumo de peixe apresente mais benefícios do que o uso de suplementos de óleo de peixe e é necessário cautela ao recomendar o seu uso. ${ }^{29}$ Deve-se sempre dar preferência ao consumo do alimento, através da ingestão de duas porções por semana dos peixes mais oleosos, tendo como base uma dieta de 2.000 kcal.9, 29

\section{Vegetais e frutas}

Em ensaios clínicos randomizados, dietas que enfatizam o consumo de frutas e vegetais favorecem a melhora de diversos fatores de risco, incluindo pressão arterial, perfil lipídico, resistência à insulina, marcadores inflamatórios, função endotelial e controle de peso. O consumo de frutas e vegetais parece reduzir o aparecimento de doenças crônicas não transmissíveis, mais especificamente, doença coronariana, devido a existência de nutrientes como potássio, folato, vitaminas, fibras e outros compostos fenólicos. Esses nutrientes atuam em diferentes mecanismos, como na redução do estresse oxidativo, na melhora no perfil de lipoproteínas, na redução da pressão arterial, no aumento da sensibilidade a insulina e na melhora da regulação da homeostase. Além disso, há uma associação inversa entre o consumo de frutas e vegetais e doença arterial coronariana, o que sugere que o consumo de tais alimentos possa ser um fator protetor contra a ocorrência de eventos cardiovasculares. O risco de doença coronariana diminui 4\% para cada porção adicional por dia de fruta e vegetais e $7 \%$ para o consumo de fruta. ${ }^{30}$

Dois grandes estudos buscaram analisar o efeito de suplementos multivitamínicos sobre DCV, o Sup-plementation in Vitamins and Mineral AntioXidants
Study (SU.VI.MAX), o estudo sobre a suplementação de antioxidantes em vitaminas e minerais antioxidantes, e o The Physicians' Health Study II (PHS-II), estudo sobre a saúde dos médicos. Tanto SU.VI.MAX quanto PHS-II não conseguiram demonstrar que os suplementos analisados possuem efeitos sobre todas as causas de mortalidade após 7,5 e 11,2 anos de acompanhamento, respectivamente. ${ }^{23,31}$ Uma explicação para estes resultados pode estar no fato que vitaminas e outros suplementos antioxidantes, que atuam em diferentes sistemas biológicos, são tão complexos, que os efeitos da suplementação com apenas um ou dois componentes é geralmente ineficaz ou traz algum malefício. Sendo assim, ainda não há razão para estimular o consumo de suplementos como forma de obter estes nutrientes, a não ser para segmentos específicos da população, como idosos por exemplo, e havendo a necessidade para tal. Do contrário, os alimentos ainda são as fontes preferenciais para que o adequado aporte de micronutrientes seja obtido. ${ }^{32}$

\section{Castanhas}

As castanhas compõem um grupo de frutos secos com casca rígida que inclui amêndoa, nozes, castanhade-caju, pecan, macadâmica, pinha, pistache, avelã, amendoim e castanha-do-brasil. Estudos têm demonstrado a relação inversa entre o consumo de castanhas e as DCV. ${ }^{33}$ Os quatro maiores estudos de coortes dos EUA: o Adventist Health Study (estudo adventista de saúde) o Iowa Women's Health Study, (estudo sobre a saúde das mulheres de Iowa), Nurses' Health Study, (estudo de saúde das enfermeiras) e o Physicians' Health Study (pesquisa sobre a saúde dos médicos) demonstraram o efeito dose-resposta entre o consumo de castanhas e a redução do risco de morte por DAC. Quando analisados em conjunto, foi possível observar uma redução média de 37\% do risco de morte por DAC (RR: 0,63; IC95\% $0,51-0,83$ ) ou ainda uma redução de $8,3 \%$ do risco de morte por DAC para cada porção semanal de castanha consumida. $^{34}$

Dentre o grupo das castanhas, a castanha-do-brasil (Bertholletia excelsa) se destaca por ser o alimento com maior teor de selênio (Se) na sua composição. ${ }^{35}$ O Se é um componente chave das selenoproteínas envolvidas em funções enzimáticas antioxidantes, anti-inflamatórias e no metabolismo dos hormônios tireoidianos. ${ }^{36}$ O Se pode ser protetor contra a doença cardiovascular e essa hipótese se baseia na habilidade da Glutationa Peroxidase (GPx), uma selenoproteína, em combater a modificação oxidativa dos lipídeos e 
reduzir a agregação plaquetária. ${ }^{36}$ Um estudo realizado na Nova Zelândia mostrou que o consumo de 2 unidades de castanha-do-brasil ao final de 12 semanas, foi capaz de aumentar $64,2 \%$ a concentração plasmática de selênio, 8,3\% a GPx plasmática e 13,2\% a GPx no sangue integral ${ }^{37}$, demonstrando o efeito sinérgico do alimento comparado ao nutriente isolado.

Contudo, alguns estudos observacionais que investigaram a associação entre o estado nutricional de Se e o risco de DCV em diferentes populações, sugerem uma possível associação no formato de curva U, onde valores baixos e valores muito altos são deletérios. ${ }^{38}$ Portanto, a suplementação de selênio não é indicada para indivíduos com estado nutricional adequado de Se (Se plasmático entre 90 e $122 \mu \mathrm{g} / \mathrm{L}){ }^{39}$

\section{A relação dos ultraprocessados com doenças crônicas e o surgimento de um padrão alimentar globalizado}

A relação entre o aumento na produção e consumo de alimentos e bebidas industrializados e a atual pandemia de obesidade e doenças crônicas em todo o mundo já foi descrita em importantes diretrizes de órgãos internacionais. Dentro deste quadro, os alimentos ultraprocessados exercem papel de destaque em função do seu alto conteúdo em sódio, açúcar, gordura saturada e trans, e alta densidade calórica. O pesquisador Carlos Augusto Monteiro sugeriu uma nova classificação dos alimentos baseada no grau de extensão e objetivo do processamento: (a) alimentos não processados ou minimamente processados; (b) ingredientes culinários processados e (c) alimentos ultraprocessados. Resumidamente, fazem parte do primeiro grupo os alimentos que sofrem um processamento mínimo e predominante físico, isto é, limpeza, porcionamento, congelamento, pasteurização, entre outros métodos que tenham como objetivo torná-los mais seguros e acessíveis ao consumidor. Temos como exemplo deste grupo as carnes frescas, grãos, legumes, verduras, frutas, leite, leguminosas e oleaginosas. A segunda classificação inclui as substâncias extraídas dos alimentos não processados ou minimamente processados. Elas sofrem um processamento físico e também químico como hidrogenação, refino, hidrólise, entre outros. Como resultado, obtêmse ingredientes culinários como amido e farinhas, óleos, sal, açúcar, xarope de milho, os quais são vendidos às indústrias ou diretamente ao consumidor final. Por fim, o processamento industrial de alimentos dos grupos 1 e 2, que leva ao surgimento de produtos destinados a ter alta durabilidade, palatabilidade, acessibilidade e conveniência durante consumo. São aqueles produtos prontos para consumir ou prontos para aquecer, que podem ser facilmente transportados e comercializados. Deste grupo fazem parte uma grande variedade de itens como barrinhas de cereais, salgadinhos, biscoitos, sorvetes e doces, cereais matinais, bebidas açucaradas, carnes processadas, alimentos enlatados e desidratados. ${ }^{40}$

Analisando os dados de três Pesquisas de Orçamento Familiar realizadas pelo Instituto Brasileiro de Geografia e Estatística(IBGE) entre março de 1987 e fevereiro de 1988, outubro de 1995 a setembro de 1996, e de julho de 2002 a junho de 2003, em 11 áreas metropolitanas do Brasil, observou-se que o consumo de alimentos não processados ou minimamente processados (grupo 1) e também de ingredientes culinários (grupo 2) vem sendo substituído pelo consumo de alimentos ultraprocessados, tanto em domicílios de baixa quanto nos de alta renda. ${ }^{41}$ Desde o início dos anos 80 , as grandes empresas multinacionais que controlam a produção e distribuição de produtos ultraprocessados em todo o mundo, e cada vez mais em países da África, Ásia e América Latina, contribuem para o processo crescente de substituição do padrão alimentar local por um padrão alimentar globalizado. Para essas grandes corporações, a mudança para mercados emergentes é necessária, uma vez que em países como Canadá, EUA e do Reino Unido, já foi atingido o ponto de saturação dos mercados locais com produtos ultraprocessados. No Brasil, ainda que haja um crescimento evidente no consumo destes produtos, há um grande potencial a ser explorado. ${ }^{42}$

O Guia Alimentar para População Brasileira de 2014 foi bastante enfático ao apontar os malefícios associados ao consumo exacerbado dos ultraprocessados. Além da composição nutricional desbalanceada e que favorece o surgimento de doenças do coração, sobrepeso e obesidade, diabetes e vários tipos de câncer, os alimentos ultraprocessados tendem a afetar de forma negativa a cultura, a vida social e o ambiente. $\mathrm{O}$ consumo crescente destes produtos desestimula o interesse dos mais jovens pela cultura alimentar tradicional de seu povo, torna o preparo do alimento e o ato de compartilhar uma refeição à mesa algo desnecessário e impacta de forma significativa o meio ambiente. Alimentos in natura ou minimamente processados, em grande variedade e predominantemente de origem vegetal, são a base de uma alimentação nutricionalmente balanceada e culturalmente apropriada. ${ }^{43}$

\section{Conclusão}

Mais importante do que excluir um determinado 


\section{Artigo de revisão}

alimento da dieta, melhorar o padrão alimentar mostrase como a mais atual tendência em relação à proteção cardiovascular. Dietas como a DASH e do Mediterrâneo já foram extensivamente estudadas e têm seus efeitos cardioprotetores comprovados. Entretanto, o grande desafio dos pesquisadores neste campo tem sido a adaptação destes padrões aos diferentes hábitos alimentares. Resultados preliminares da tentativa de adaptação do padrão mediterrâneo à cultura alimentar da população brasileira foram animadores e mostram que é possível reduzir fatores de risco cardiovascular com alimentos nacionais e acessíveis. Dentro deste processo, combater o crescente consumo de alimentos ultraprocessados entre a população brasileira é fundamental e tal orientação deve ser enfatizada por todos os profissionais da nutrição. Desta forma, será possível atuar de forma ativa na preservação do padrão alimentar tradicional do brasileiro, bem como na prevenção de doenças cardiovasculares e outras patologias.

\section{Referências}

1. Mendis S, Puska P, Norrving, B. Global Atlas on cardiovascular disease prevention and control. Geneva: World Health Organization, 2011.

2. Mansur AdP, Favarato, D. Mortalidade por Doenças Cardiovasculares no Brasil e na Região Metropolitana de São Paulo:Atualização 2011. Arq Bras Cardiol. 2012;99(2):755-61.

3. Simao AF, Precoma DB, Andrade JP, et al. [In Process Citation]. Arq Bras Cardiol.101(6 Suppl 2):1-63.

4. Konstantinov IE, Jankovic GM. Alexander I. Ignatowski: a pioneer in the study of atherosclerosis. Tex Heart Inst J.2013;40(3):246-9.

5. Go AS, Mozaffarian D, Roger VL, Benjamin EJ, et al. Heart disease and stroke statistics--2014 update: a report from the American Heart Association. Circulation.2014;129(3):e28-e292.

6. Page IH, Stare FJ, Corcoran AC, et al. Atherosclerosis and the fat content of the diet. J Am Med Assoc. 1957;164(18):2048-51.

7. Tracy SW. Something new under the sun? The Mediterranean diet and cardiovascular health. N Engl J Med. 2013;368(14):1274-6.

8. Eckel RH, Jakicic JM, Ard JD, et al. 2013 AHA/ACC guideline on lifestyle management to reduce cardiovascular risk: a report of the American College of Cardiology/American Heart Association Task Force on Practice Guidelines. Circulation.2013;129(25 Suppl 2):S76-99.

9. Mozaffarian D, Appel LJ, Van Horn L. Components of a cardioprotective diet: new insights. Circulation.2011;123(24):2870-91.

10. Salehi-Abargouei A, Maghsoudi Z, Shirani F,et al. Effects of Dietary Approaches to Stop Hypertension (DASH)-style diet on fatal or nonfatal cardiovascular diseases--incidence: a systematic review and meta-analysis on observational prospective studies. Nutrition.2013;29(4):611-8.

11. Trichopoulou A, Bamia C, Norat T, et al. Modified Mediterranean diet and survival after myocardial infarction: the EPIC-Elderly study. Eur J Epidemiol. 2007;22(12):871-81.

12. Stone NJ, Robinson JG, Lichtenstein AH, et al. 2013 ACC/AHA guideline on the treatment of blood cholesterol to reduce atherosclerotic cardiovascular risk in adults: a report of the American College of Cardiology/American Heart Association Task Force on Practice Guidelines. J Am Coll Cardiol.2013;63(25 Pt B):2889-934.

13. de Lorgeril M, Salen P, Martin JL,et al. Mediterranean diet, traditional risk factors, and the rate of cardiovascular complications after myocardial infarction: final report of the Lyon Diet Heart Study. Circulation. 1999;99(6):779-85.

14. Knoops KT, de Groot LC, Kromhout D, et al. Mediterranean diet, lifestyle factors, and 10-year mortality in elderly European men and women: the HALE project. JAMA. 2004;292(12):1433-9.

15. Mitrou PN, Kipnis V, Thiebaut AC, et al. Mediterranean dietary pattern and prediction of all-cause mortality in a US population: results from the NIH-AARP Diet and Health Study. Arch Intern Med. 2007;167(22):2461-8.

16. Fung TT, Rexrode KM, Mantzoros CS,et al. Mediterranean diet and incidence of and mortality from coronary heart disease and stroke in women. Circulation. 2009;119(8):1093-100.

17. Sofi F, Abbate R, Gensini GF, Casini A. Accruing evidence on benefits of adherence to the Mediterranean diet on health: an updated systematic review and meta-analysis. Am J Clin Nutr.2010;92(5):1189-96.

18. Estruch R, Ros E, Salas-Salvado J, et al. Primary prevention of cardiovascular disease with a Mediterranean diet. N Engl J Med.2013;368(14):1279-90.

19. Bach-Faig A, Berry EM, Lairon D, et al. Mediterranean diet pyramid today. Science and cultural updates. Public Health Nutr.2011;14(12A):2274-84.

20. Bellisle F. Infrequently asked questions about the Mediterranean diet. Public Health Nutr. 2009;12(9A):1644-7.

21. Weber B, Galante AP, Bersch-Ferreira AC, et al. Effects of Brazilian Cardioprotective Diet Program on risk factors in patients with coronary heart disease: a Brazilian Cardioprotective Diet randomized pilot trial. Clinics (Sao Paulo).2012;67(12):1407-14.

22. Mozaffarian D, Rimm EB. Fish intake, contaminants, and human health: evaluating the risks and the benefits. JAMA. 2006;296(15):1885-99.

23. Hercberg S, Galan P, Preziosi P, et al. The SU.VI.MAX Study: a randomized, placebo-controlled trial of the health effects of antioxidant vitamins and minerals. Arch Intern Med. 2004;164(21):2335-42.

24. Yokoyama M, Origasa $H$, Matsuzaki M, et al. Effects of eicosapentaenoic acid on major coronary events in hypercholesterolaemic patients (JELIS): a randomised open-label, blinded endpoint analysis. Lancet. 2007;369(9567):1090-8.

25. Rizos EC, Ntzani EE,Bika E, et al.. Association Between Omega-3 Fatty Acid Supplementation and Risk of Major Cardiovascular Disease Events A Systematic Review and Meta-analysis. JAMA. 2012;308(10).

26. Bucher HC, Hengstler P, Schindler C, et al. N-3 polyunsaturated fatty acids in coronary heart disease: a meta-analysis of randomized controlled trials. Am J Med. 2002;112(4):298-304.

27. He K, Rimm EB, Merchant A, et al. Fish consumption and risk of stroke in men. JAMA. 2002;288(24):3130-6.

28. Hansen JC, Pedersen HS, Mulvad G. Fatty acids and antioxidants in the Inuit diet. Their role in ischemic heart disease (IHD) and possible interactions with other dietary factors. A review. Arctic Med Res. 1994;53(1):4-17.

29. He K. Fish, long-chain omega-3 polyunsaturated fatty acids and prevention of cardiovascular disease--eat fish or take fish oil supplement? Prog Cardiovasc Dis. 2009;52(2):95-114. 
Mariana R. C. Portugal e cols. • Dos alimentos cardioprotetores ao padrão alimentar: uma revisão da literatura

30. Dauchet L, Amouyel P, Hercberg S, et al. Fruit and vegetable consumption and risk of coronary heart disease: a meta-analysis of cohort studies. J Nutr. 2006;136(10):2588-93.

31. Christen WG, Gaziano JM, Hennekens CH. Design of Physicians' Health Study II--a randomized trial of beta-carotene, vitamins $\mathrm{E}$ and $\mathrm{C}$, and multivitamins, in prevention of cancer, cardiovascular disease, and eye disease, and review of results of completed trials. Ann Epidemiol. 2000;10(2):125-34.

32. Lichtenstein AH, Russell RM. Essential nutrients: food or supplements? Where should the emphasis be? JAMA. 2005;294(3):351-8.

33. Mozaffarian D, Appel LJ, Van Horn L. Components of a Cardioprotective Diet: New Insights. Circulation. 2011;123(24):2870-91.

34. Sabaté J, Ang Y. Nuts and health outcomes: new epidemiologic evidence. The American Journal of Clinical Nutrition. 2009;89(5):1643S-8S.

35. Freitas SCd, Gonçalves EB, Antoniassi R, et al. Meta-analysis of selenium content in Brazil nuts. Brazilian Journal of Food Technology. 2008;11(1):54-62.

36. Rayman MP. The importance of selenium to human health. The Lancet. 2000;356(9225):233-41.
37. Thomson CD, Chisholm A, McLachlan SK, et al. Brazil nuts: an effective way to improve selenium status. Am J Clin Nutr. 2008;87(2):379-84.

38. Stranges S, Navas-Acien A, Rayman MP, et al. Selenium status and cardiometabolic health: state of the evidence. Nutrition, metabolism, and cardiovascular diseases : NMCD. 2010;20(10):754-60.

39. Rayman MP. Selenium and human health. Lancet. 2012;379(9822):1256-68.

40. Monteiro CA, Levy RB, Claro RM, et al. A new classification of foods based on the extent and purpose of their processing. Cad Saude Publica.2010;26(11):2039-49.

41. Monteiro CA, Levy RB, Claro RM,et al. Increasing consumption of ultra-processed foods and likely impact on human health: evidence from Brazil. Public Health Nutr.2011;14(1):5-13.

42. Monteiro CA, Cannon G. The impact of transnational "big food" companies on the South: a view from Brazil. PLoS Med.2012;9(7):e1001252.

43. Saúde Md. Guia Alimentar da População Brasileira. In: Básica DdA, editor. 2 ed. Brasília-DF: Ministério da Saúde; 2014. p. 156. 